Revista Brasileira de Cartografia

ISSN 1808-0936 | https://doi.org/10.14393/revbrascartogr

Sociedade Brasileira de Cartografia, Geodésia, Fotogrametria e Sensoriamento Remoto

\title{
Modelagem Geométrica de Fachadas usando Nuvens de Pontos LiDAR
}

\section{Geometric Facade Modeling using LiDAR Point Clouds}

\author{
Luís Antônio Soares e Sousa ${ }^{1}$, Jorge Antônio Silva Centeno ${ }^{2}$
}

1 Universidade Federal do Paraná, Programa de Pós-Graduação em Ciências Geodésicas, Curitiba, Brasil. lassousa21@gmail.com ORCID: https://orcid.org/0000-0003-4494-8808

2 Universidade Federal do Paraná, Programa de Pós-Graduação em Ciências Geodésicas, Curitiba, Brasil. centeno@ufpr.br ORCID: https://orcid.org/0000-0002-2669-7147

Resumo: LiDAR se mostrou valioso na análise do meio urbano, pois permite a captura de informações tridimensionais para detectar e modelar edifícios. O desenvolvimento do LiDAR móvel terrestre abriu novas possibilidades para a modelagem 3D em áreas urbanas. Neste artigo é apresentada uma metodologia semiautomática que visa calcular modelos tridimensionais de edifícios a partir de nuvens de pontos obtidas com LiDAR terrestres móveis. Para tanto, a nuvem de pontos é segmentada em blocos de planos uniformes analisando a variação da densidade de pontos ao longo das principais direções da fachada. Isso permite segmentar a nuvem de pontos em regiões planas que posteriormente são combinadas para construir o modelo $3 \mathrm{D}$, mesmo quando a fachada possui diferentes planos paralelos. O principal diferencial da metodologia é a utilização dos histogramas de frequência para segmentar a nuvem de pontos e detectar as bordas da fachada, garantindo economia de tempo em relação aos métodos tradicionais.

Palavras-chave: Modelagem de Edificações. Modelagem de fachadas. Modelagem de Telhados. LiDAR. Segmentação de nuvem de pontos.

\begin{abstract}
LiDAR has established itself as a valuable technology in urban analysis, enabling capturing threedimensional information to detect and model buildings. The development of mobile terrestrial LiDAR opened new possibilities for 3D modelling in urban areas. In this paper it is introduced a semiautomatic methodology that aims at computing three-dimensional models of buildings from mobile terrestrial LiDAR point clouds. For this purpose, the point cloud is segmented into uniform planes blocks analyzing the variation of the point density along the main directions of the façade. This enables segmenting the point cloud in plane regions that are later combined to build up the 3D model, even when the façade has different parallel planes. The main differential of the methodology is the use of the frequency histograms to segment the point cloud and detect the borders on the facade, guaranteeing time savings compared to traditional methods.
\end{abstract}

Keywords: Building Modeling. Facade Modeling. Roof Modeling. LiDAR. Cloud Points Segmentation.

\section{INTRODUÇÃO}

A documentação da geometria de edifícios existentes consiste em medir e armazenar suas informações a respeito de suas dimensões e materiais para modelar, interpretar e materializar a ideia do projeto original. Devido ao alto índice de detalhes, torna-se um desafio desenvolver metodologias eficientes que possam ser utilizadas no processo de análise, identificação e extração das características geométricas de prédios e que possam ser generalizadas às distintas edificações. Os primeiros levantamentos desta natureza eram executados por medição direta, utilizando trena ou teodolito, tendo como vantagem a simplicidade do processo e desvantagens a morosidade, principalmente quando realizadas em monumentos ou sítios históricos com muitos detalhes. Com o surgimento de novas tecnologias na engenharia, como o levantamento de densas nuvens de pontos com o LiDAR (Ligth Detection and Ranging), a maneira de medir e documentar a forma de um objeto passou por grandes modificações. A disponibilidade de uma grande quantidade de pontos, em lugar de alguns pontos selecionados criteriosamente, transformou o processo de medição. 
Grande parte dos primeiros estudos relativos à modelagem de edificações com dados LiDAR se concentrou nos telhados, pois era comum usar sensores aerotransportados. A integração de dados LiDAR com ortofotos, mapas existentes e imagens de satélite também foi vastamente pesquisada. Por exemplo, Vosselman e Dijkman (2001) utilizaram a combinação de dados LiDAR com plantas baixas de edificações, enquanto Rottensteiner et al. (2004) e Awrangjeb et al. (2012) combinaram dados LiDAR e imagens aéreas.

As abordagens metodológicas propostas para a modelagem de telhados também variam. Rottensteiner (2003) propôs detectar planos na nuvem de pontos e depois buscar a interseção de planos para modelar o telhado. Outros autores propuseram aplicar métodos de crescimento de regiões em uma estrutura TIN (SAMPATH; SHAN, 2010) ou em uma grade raster (JOCHEM et al., 2012). Lafarge et al. (2010) e Orthuber e Avbelj (2015) criaram um banco de dados de formas de telhados e verificaram o grau de semelhança da nuvem de pontos medida com esses modelos geométricos. Kim e Shan (2011) focaram na análise de arestas Kabolizade et al. (2012) e Sabariego (2018) utilizaram dados LiDAR aplicando algoritmos genéticos.

As aplicações destes modelos são várias, como, por exemplo: Cadastro técnico (Cadastro 3D) (FONSECA et al., 2016); Expansão, planejamento e política urbana (FERREIRA, 2014); Estudos de potencial fotovoltaico e coleta de água pluvial (AMISSE, 2016) e fins arquitetônicos/BIM (SOUZA et al., 2015); DEZEN-KEMPTER et al., 2015). Devido à carência de dados das paredes dos prédios, os primeiros estudos combinaram a geometria obtida de destas nuvens de pontos com texturas de fotografias, para produzir modelos tridimensionais de uma edificação, como é mostrado em Frueh et al. (2004).

Com o surgimento dos sistemas LiDAR terrestres e móveis foi possível obter maior detalhamento das fachadas, o que gerou uma nova onda de propostas metodológicas. Por exemplo, Yang et. al. (2013) propuseram um método de extração semiautomática de footprints das fachadas de edifícios, a paetir de nuvens de pontos obtidas por um sensor LiDAR instalado em um veículo (mapeamento móvel). Zhang (2018) desenvolveu um algoritmo misto para a extração de fachadas de edifícios a partir de nuvens de pontos obtidas com LiDAR terrestre. Pu e Vosselman (2009) propuseram uma metodologia para a modelagem semiautomática de fachadas usando dados LiDAR e imagens fotográficas.

Quando a varredura LiDAR tem alta densidade de pontos é possível também detectar e modelar feições e detalhes presentes nas fachadas, melhorando sua representação e análise. Por exemplo, Belton e Lichti (2006) desenvolveram um método para classificar os pontos que definem as feições de fachadas com base na orientação local da nuvem de pontos, calculada a partir de autovetores da matriz variância-covariância de uma vizinhança na nuvem de pontos. Da mesma maneira, Briese e Pfeifer (2008) propuseram uma abordagem para a modelagem de edificações baseada principalmente na detecção de características lineares na nuvem de pontos.

O levantamento LiDAR aerotransportado permite a modelagem dos telhados, porém não das paredes ou fachadas. Por outro lado, o LiDAR terrestre móvel mostrou-se eficiente no mapeamento das paredes e fachadas, mas impossibilitado de captar dados dos telhados. Uma solução viável para se obter a representação completa de um edifício é a combinação de levantamentos aerotransportado e terrestre. Estas informações são complementares e, consequentemente, facilitam a obtenção de modelos mais fiéis à realidade (BOULAASSAL et al., 2007) sem a necessidade de coletas manuais diretas sobre a construção. O resultado ainda pode ser utilizado como fonte para análises BIM (Building Information Model), tornando as etapas de uma obra, seja de planejamento, execução ou pós-obra, mais realistas e com maior confiabilidade.

Considerando que a modelagem de telhados é um tópico bastante explorado na comunidade científica, este trabalho se concentra no desenvolvimento de métodos para gerar a representação de fachadas, de forma semiautomática, com a finalidade de compor modelos tridimensionais detalhados, que possam ser utilizados como subsídios para documentação do patrimônio e definição de planos de gestão, bem como suporte para planejamento de obras e melhorias.

\section{MATERIAIS E MÉTODO}

Os materiais e o método que serão utilizados para a realização do trabalho, visando a modelagem tridimensional de edificações, são apresentados a seguir. 


\subsection{Materiais}

A área de estudo encontra-se no campus do Centro Politécnico da Universidade Federal do Paraná (UFPR). Os dados utilizados foram adquiridos em diferentes intervalos de tempo e por dois métodos distintos: um levantamento aéreo e outro terrestre.

O aerolevantamento LIDAR (Figura 1A) foi realizado em agosto de 2012, na cidade de Curitiba, utilizando o sensor Pegasus HD500 e disponibilizado pelo Instituto de Tecnologia para o Desenvolvimento Lactec. O levantamento terrestre (Figura 1B) foi feito em abril de 2014, utilizando o Sistema Laser Terrestre Móvel (MTLS) Suíço Pegasus One, pertencente a empresa Leica Geosystem. Os dados foram cedidos pela empresa Esteio Engenharia e Aerolevantamentos S.A para a UFPR para fins acadêmicos.

Figura 1 - Recorte inicial da Nuvem de pontos do Sistema Pegasus HD500 (A) e do Sistema Laser Terrestre Móvel
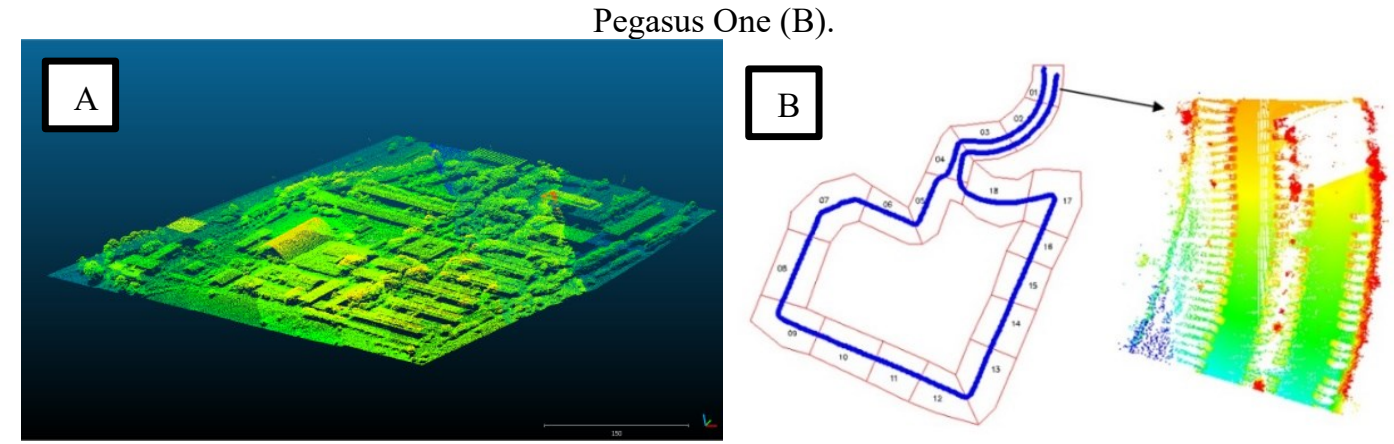

Fonte: (A) O autor (2021); (B) Peixoto (2016).

O conjunto de bibliotecas e softwares usados para o desenvolvimento deste trabalho foram: I. Microcomputador desktop com processador Intel CoreTM Duo CPU, 4 Gb de RAM e 260 Gb de espaço em disco; II. LiDAR Mapping Suite (LMS) para extração e processamento dos dados brutos do ATML Pegasus HD500; III. MicroStation para executar a funcionalidade TerraSolid com o pacote de módulos TerraScan utilizado para visualização da nuvem de pontos, consolidação das nuvens de pontos únicas, filtragem das nuvens de pontos, recorte de regiões e classificação da nuvem de pontos; IV. Sketchup para modelagem tridimensional da edificação e V. Os equipamentos e softwares utilizados no processamento da nuvem de pontos do TLS podem ser encontrados em Peixoto (2016).

\subsection{Método}

A modelagem de um prédio, neste estudo, se concentra no detalhamento da geometria de suas fachadas, com base em um levantamento LiDAR terrestre móvel. A metodologia utilizada se divide em quatro etapas: o pré-processamento; a separação da nuvem de pontos em planos; a modelagem destes planos isoladamente; e a integração dos resultados em um único modelo tridimensional.

A hipótese básica da metodologia é que uma fachada é composta por elementos regulares, orientados principalmente nas direções paralelas às bordas da fachada. Esta situação se verifica na maioria das construções e um exemplo pode ser visto na figura 2A. Porém, a liberdade em termos de projeto arquitetônico pode resultar em situações que fogem a esta orientação, como no exemplo da figura 2B. O segundo exemplo mostra uma distribuição irregular de janelas, mesmo que suas formas sejam paralelas aos eixos principais do plano da fachada. Em termos práticos, estas variações são raras, devido às dificuldades na construção. 
Figura 2 - Diferentes arranjos de elementos de uma fachada: (A) Fachada regular ou (B) irregular.
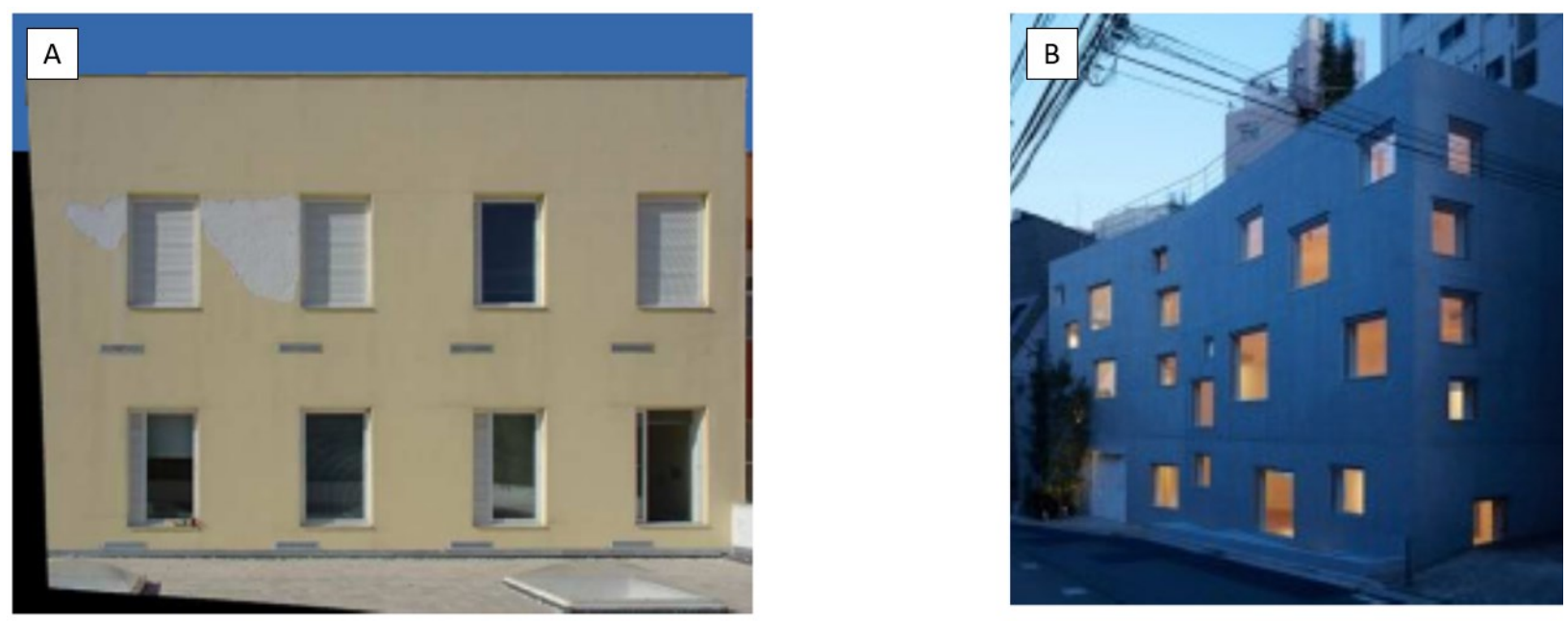

Fonte: (A) Tsironis et al. 2017; (B) Florian Busch (2019).

O princípio comum às diferentes etapas desta metodologia é a análise do histograma de frequência da nuvem de pontos projetada nas direções paralelas ao plano da parede. Isto pode ser entendido com ajuda da figura 3, onde é mostrado um exemplo ilustrativo da varredura de uma fachada, parecida àquela mostrada na figura 2A. Os pontos em preto representam os locais onde o pulso LiDAR foi refletido pela superfície da fachada. No caso das janelas, assume-se, por enquanto, que elas são transparentes e deixaram passar o pulso, que foi assim perdido. Na mesma figura são mostrados dois histogramas, um horizontal (ao longo do eixo x) e outro vertical (eixo y). Para a construção destes histogramas é contado o número de ocorrências de pontos ao longo de cada eixo, usando um passo pequeno para definir as barras do histograma. O tamanho do passo deve ser compatível com a densidade da varredura e com a acurácia almejada.

Figura 3 - Exemplo de histogramas da nuvem de pontos em direções determinadas.

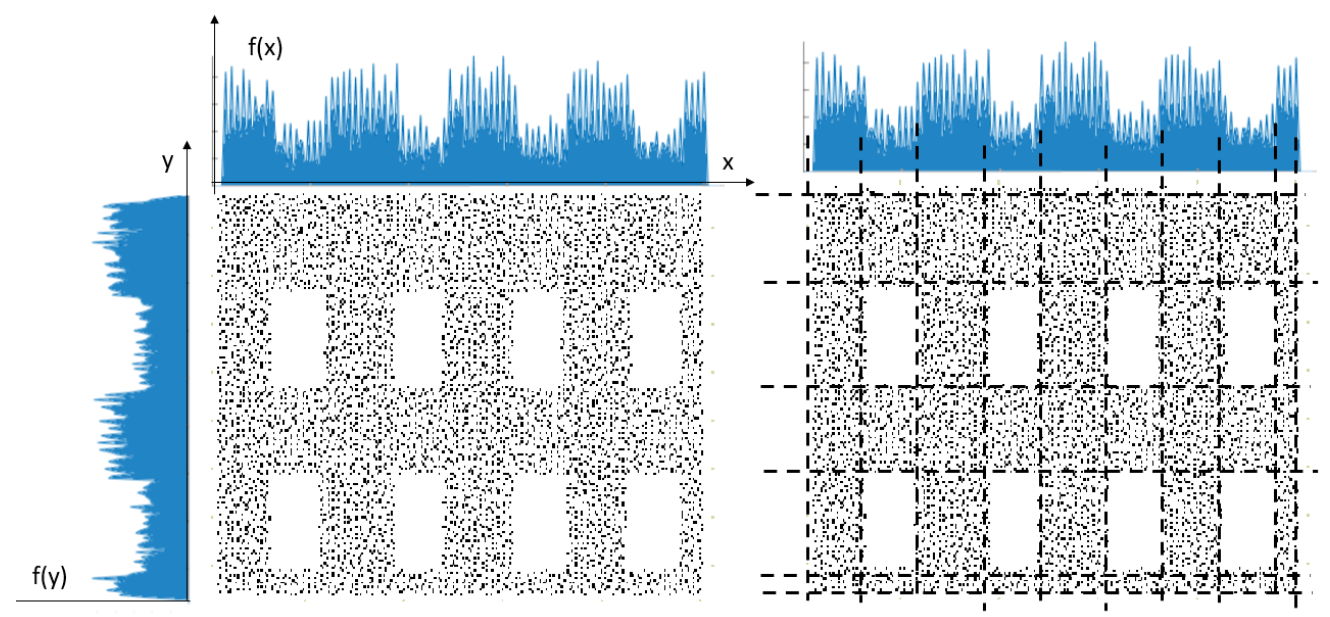

Fonte: O autor (2021).

Tomado como exemplo o histograma ao longo de X (horizontal), nota-se que valores menores ocorrem nos trechos onde estão localizadas as janelas. Localizando as transições entre valores altos e baixos no histograma poder-se-ia determinar o local onde se localizam as janelas e assim derivar uma descrição da fachada. A aplicação desta ideia, a princípio simples, requer outras etapas de processamento, que serão detalhadas a seguir.

A metodologia proposta, mostrada na Figura 4, apresenta várias etapas antes de seguir para a segmentação propriamente dita. Os passos são a orientação da fachada, a remoção de elementos espúrios, como a vegetação, a decomposição da fachada em planos com diferentes profundidades, a modelagem das janelas e 
portas em cada plano e, finalmente, a composição dos elementos segmentados em um modelo 3D. Estas etapas necessitam de uma atenção maior, logo estão detalhadas a seguir.

Figura 4 - Fluxograma da metodologia para modelagem de fachadas.

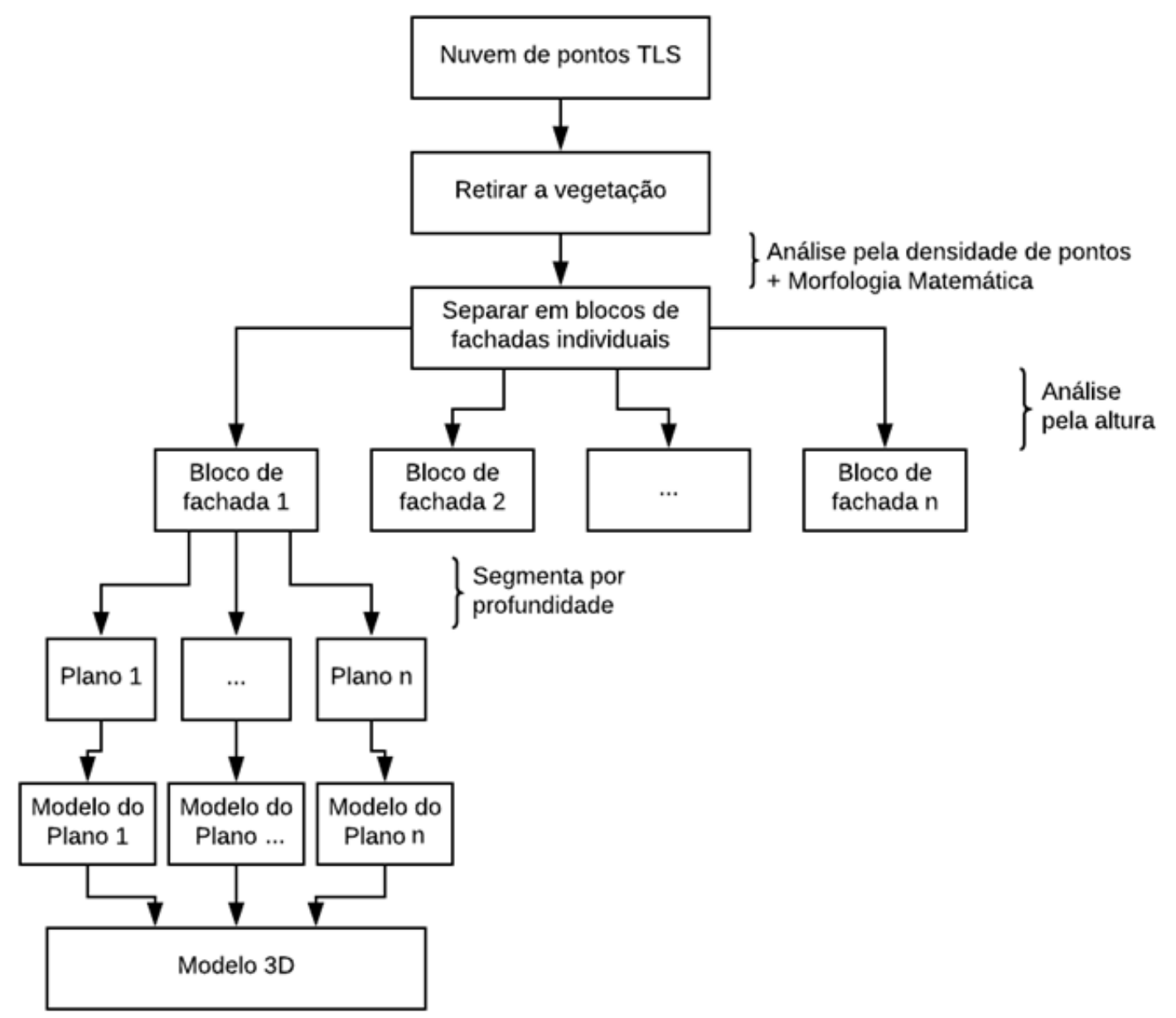

Fonte: $\mathrm{O}$ autor (2021).

\subsubsection{PRÉ-PROCESSAMENTO (ORIENTAÇÃO)}

A região de estudo já tinha sido objeto de um levantamento com LiDAR terrestre móvel (TLS) e os dados foram cedidos para esta pesquisa. O levantamento foi planejado para ter densidade média de projeto de 2400 pontos por $\mathrm{m}^{2}$, embora esta grandeza varie em função da distância entre as superfícies e o sensor. Deste conjunto de dados foram extraídos recortes de forma manual, utilizando o software CloudCompare.

O resultado dos recortes se aproxima de um plano vertical, representando as fachadas de um bloco/edifício. Para facilitar a manipulação dos dados, a nuvem de pontos foi orientada de forma a torná-la perpendicular em relação ao sistema de coordenadas (coordenada X) do Laser Scanner Terrestre. Para isto, calcula-se o vetor de médias da nuvem de pontos e a matriz variância-covariância dos pontos em relação a esta referência. Os autovetores desta matriz fornecem as principais direções da dispersão dos pontos. O menor autovetor aponta na direção perpendicular ao plano médio da fachada. Esta direção será adotada como a profundidade. $\mathrm{O}$ eixo " $\mathrm{X}$ " é dado pela direção do maior autovetor; o eixo $\mathrm{Z}$ é definido como sendo o segundo autovetor (altura) e o eixo Y como o terceiro (profundidade). Com estes vetores, é composta a matriz de rotação e a transformação de corpo rígido é aplicada, como é mostrado na Eq. (1).

$$
\left[\begin{array}{l}
X \\
Y \\
Z
\end{array}\right]=\left[\begin{array}{lll}
v_{11} & v_{12} & v_{13} \\
v_{21} & v_{22} & v_{23} \\
v_{31} & v_{32} & v_{33}
\end{array}\right]\left[\begin{array}{l}
x \\
y \\
Z
\end{array}\right]
$$


onde $(x, y, Z)$ : coordenadas da varredura LiDAR; $(\mathrm{X}, \mathrm{Y}, \mathrm{Z})$ : coordenadas após a transformação; $v_{i j}$ : j-éssimo elemento do i-éssimo autovetor.

\subsubsection{REDUÇÃO DOS PONTOS RELATIVOS À VEGETAÇÃO}

Nos levantamentos do TLS há a ocorrência de outros objetos, além das fachadas, principalmente vegetação. Esses objetos devem ser eliminados para que se consiga isolar as fachadas. As paredes de uma fachada refletem quase todos os pontos incidentes, sendo assim a densidade de pontos medidos nestas regiões é bem próxima da densidade esperada para o projeto. A vegetação, pelo contrário, não reflete todos os pulsos emitidos pelo sensor, por não apresentar uma superfície efetivamente continua. Alguns pulsos a atravessam e se perdem, fazendo com que a densidade de pontos seja menor do que em edificações. Isto permite separar a vegetação das fachadas analisando a densidade de pontos. Para isso, gera-se um histograma de frequência ao longo do eixo $\mathrm{X}$, direção principal da nuvem de pontos e avalia-se a densidade linear de pontos. O passo do histograma deve ser relativamente pequeno, para não excluir partes dos prédios. Recomenda-se usar equidistâncias de $5 \mathrm{~cm}$. Os trechos com densidade de pontos menor que $90 \%$ da densidade de projeto são considerados vegetação e seus pontos removidos do conjunto.

\subsubsection{SEPARAÇÃO DE BLOCOS DE FACHADA}

Várias fachadas podem estar presentes em uma nuvem de pontos de um bloco de quadra. Se os prédios estivessem separados um do outro seria fácil identificar cada um deles, porém, na realidade, muitas vezes os prédios são adjacentes, formando um bloco contínuo, especialmente em áreas mais densamente ocupadas. As fachadas mais simples seriam retangulares, porém na realidade muitas delas são compostas por trechos com alturas diferentes. Estes fatos motivaram a dividir a nuvem de pontos em blocos de altura similar para serem analisados de forma separada.

Para definir tais blocos foram procurados locais onde ocorreram mudanças bruscas de altura, ou seja, as bordas dos blocos. Para isto é calculado o perfil discreto da nuvem de pontos ao longo do eixo X. A nuvem é então dividida em intervalos constantes ao longo da direção X e cada intervalo é representado pelo valor da cota máxima no trecho.

A fim de remover pontos espúrios presentes na cena e facilitar a identificação dos vértices da edificação, o perfil foi suavizado utilizando o operador de abertura da Morfologia Matemática (erosão seguida de dilatação). A abertura permite eliminar concentrações de pontos pequenas, em relação ao elemento estruturante, o que tem como consequência a remoção de pequenas ilhas formadas por elementos como cabos ou antenas, e a quebra de istmos, o que contribui para suavizar os contornos. Inicialmente, a erosão remove os elementos menores do conjunto e diminui o tamanho dos objetos. Depois, a dilatação expande as bordas dos objetos, se aproximando da sua forma original, mas sem a presença dos elementos menores. Deve-se salientar que a dilatação não é a operação complementar da erosão.

Após a suavização, o contorno superior da fachada é analisado, como é mostrado na figura 5. Primeiro, as coordenadas dos extremos superiores de cada retângulo, que representa o trecho no histograma, são aproximadas por linhas retas, as linhas do perfil mostrado na figura 5a. Nesse novo perfil, identifica-se pontos que marcam as variações expressivas, que caracterizam a transição entre dois blocos diferentes (figura 5b). Isto é feito analisando a variação da direção dos trechos antes e depois de cada ponto, ou seja, o ângulo formado por estes trechos. É assumido que uma borda ocorre quando o ângulo for próximo de $90^{\circ}$. A partir deste ponto, cada bloco é tratado de forma separada (Figura 5c). 
Figura 5 - Análise de um perfil longitudinal. A) Aproximação do contorno por segmentos de reta; B) detecção de bordas entre blocos; C) resultado da separação e blocos.

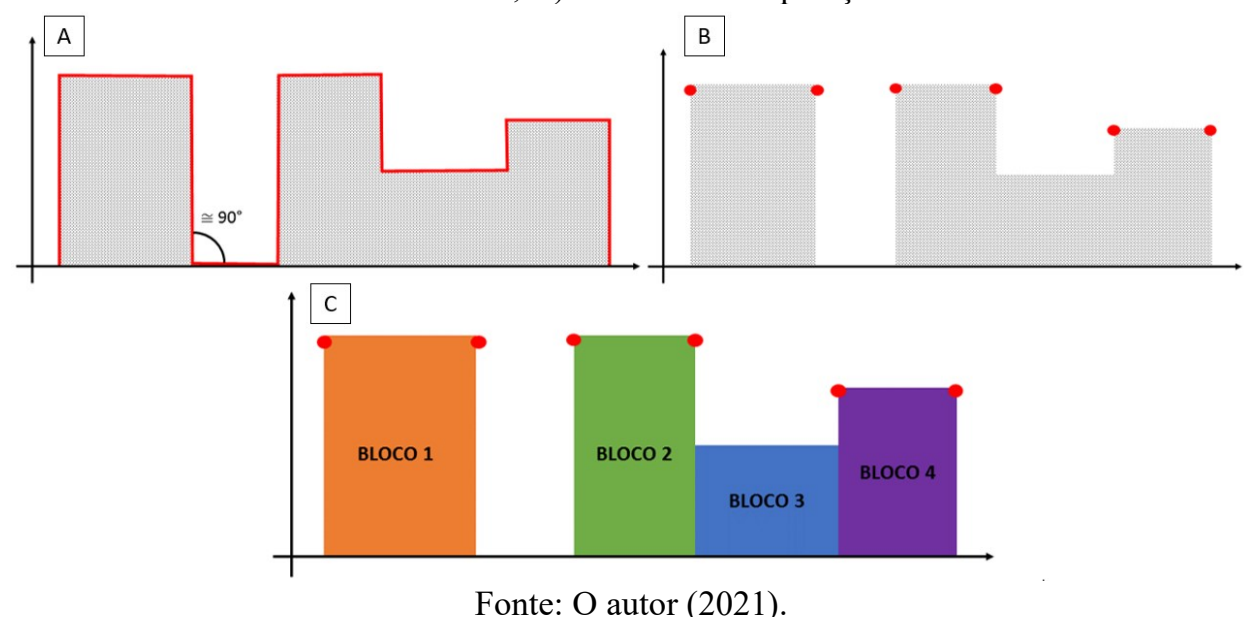

\subsubsection{SEPARAÇÃO EM PLANOS DE FACHADA}

A seguir, a variação de profundidade de cada bloco foi analisada com o intuito de verificar se ele é composto por mais de um plano. Para isto, cria-se outro histograma de frequência baseando na densidade da nuvem de pontos, mas desta vez ao longo do eixo da profundidade. Nesta etapa, caso a parede seja composta por um único plano, o histograma será unimodal. Porém, caso a parede seja composta por vários planos, os picos do histograma são considerados como os locais onde diferentes planos ocorrem em diferentes profundidades. A profundidade destes picos foi calculada e usada para classificar o restante dos pontos pelo critério da distância mínima. Assim, cada ponto foi assinado a uma profundidade, um plano. Aconselha-se o uso de um curto passo para o histograma, como $0,05 \mathrm{~m}$, a fim de obter um resultado mais detalhado de cada plano. Esta distância deve considerar a largura média de uma parede. Na Figura 6 é mostrada uma representação gráfica deste conceito. A fachada é composta por vários planos paralelos. Um plano de parede na frente (em vermelho) e outro atrás (em verde). Por fim, existe outro plano com a porta e a janela. À direita da figura mostra-se o histograma das profundidades, caracterizado por três picos, o que permite separar os planos da fachada.

Figura 6 - Geração do Histograma de Profundidade. A) fachada formada por três planos (vermelho, verde e laranja). B) histograma de profundidades desta fachada.

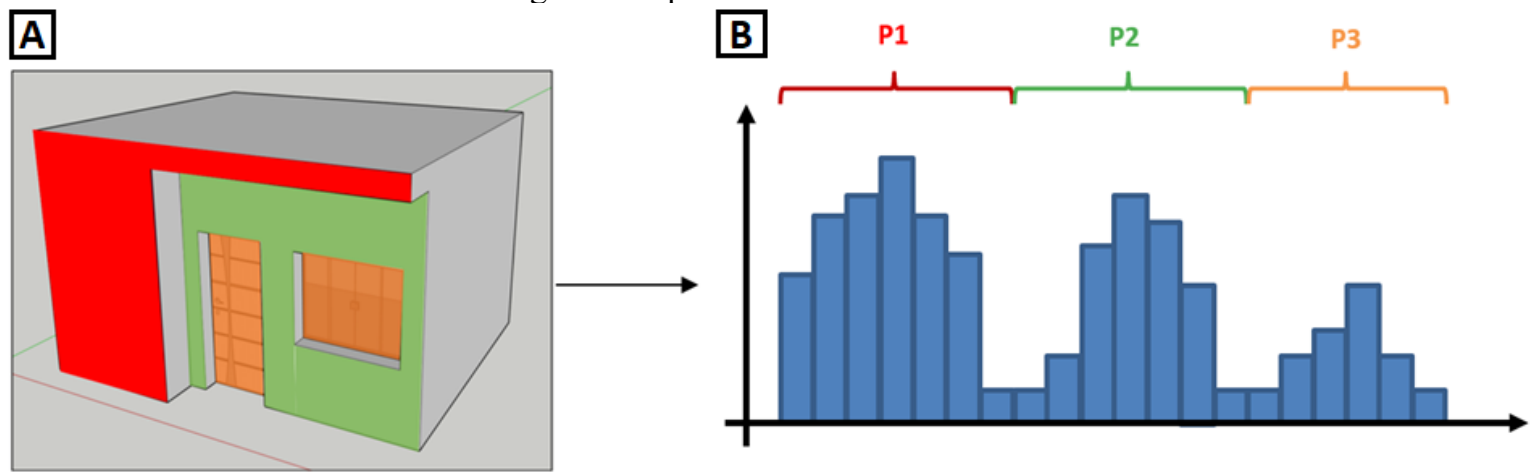

Fonte: O autor (2021).

Percebe-se que, por haver maior frequência de pontos nas paredes, devido à solidez e opacidade dos materiais que as compõe, estas regiões possuem maiores picos no histograma. Já em regiões que possuem janelas, principalmente, de vidro, há bastante dispersão de pontos, diminuindo a frequência. Logo, os picos representam cada plano e, dessa forma, os pontos correspondentes a cada grupo serão separados e analisados de forma independente. 


\subsubsection{MODELAGEM DAS FEIÇÕES PRESENTES NA FACHADA}

Nesta etapa, os pontos de cada plano, separado na etapa anterior, são analisados para formar blocos retangulares como base para a modelagem geométrica. É assumido que todos os pontos atribuídos a um plano possuem a mesma profundidade, a profundidade média do plano. Considerando este plano, ocorrem vazios na nuvem de pontos nos locais onde estão as janelas, pois os pulsos incidentes nestas regiões estarão contidos em outro plano. Com base na análise dos histogramas parciais na direção horizontal e vertical, conforme o conceito descrito na Figura 3, é possível estimar a posição das janelas uma vez isolado o respectivo plano. Uma otimização dessa abordagem consiste em calcular os histogramas apenas dos pontos nas bordas, como é descrito a seguir.

A ideia é detectar na nuvem de pontos de uma parede os pontos que caracterizam as bordas de janelas e portas. Para isto, os pontos são projetados em uma grade regular paralela ao plano e a ocorrência de pontos em cada célula é armazenada. Uma célula com valor zero mostra que nenhum ponto ocorre nesta região (vazio). Já um valor igual a um foi atribuído se pelo menos um ponto ocorre na célula. Isto permite representar a nuvem como uma imagem binária (Figura 7a). A resolução da grade deve ser compatível com a densidade de pontos esperada na parede. Caso o tamanho da célula seja menor que o espaçamento médio entre pontos definido pela resolução do levantamento, muitos locais ficarão vazios. A seguir, são identificadas as bordas nesta imagem (Figura 7b). Para isto, aplica-se o operador de erosão, da morfologia matemática, para depois calcular a diferença entre a grade original e a erodida, que fornece apenas as bordas. Um elemento estruturante retangular $3 \times 3$ é usado nesta etapa. Finalmente, separou-se apenas os pontos que ocorrem nestas fronteiras (Figura 7c).

Figura 7 - Histogramas na vertical e horizontal de pontos nas bordas.
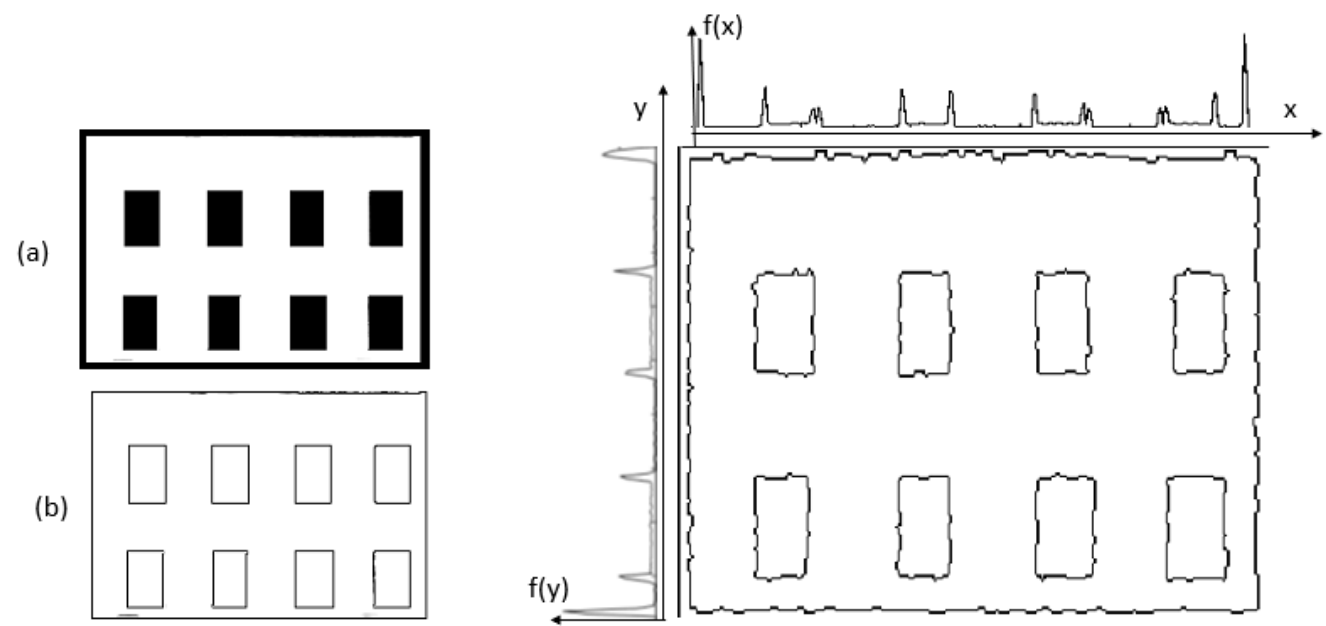

(c)

Fonte: O autor (2021).

Os histogramas deste conjunto reduzido de pontos apresentaram picos bem definidos nas regiões de fronteiras, causadas pelos limites das janelas ou portas. Assim sendo, detecta-se os picos dos histogramas para conformar uma grade irregular com regiões ocupadas por janelas e partes da parede. Ressalta-se que esta hipótese é satisfeita na maioria das construções, mas que não se trata de uma regra geral, ou seja, algumas construções não poderão ser tratadas dessa forma.

Com a localização das linhas de bordas, o plano da parede é dividido em segmentos retangulares de tamanho diferente, uma grade irregular. A seguir é calculada a densidade de pontos em cada segmento e os segmentos com baixa densidade de pontos são descartados. Para isto, a densidade de projeto é usada como referência. Finalmente, a fachada é reconstruída pela união de todos os segmentos válidos. Na etapa final, segmentos retangulares adjacentes são combinados para formar retângulos maiores e simplificar a representação. Neste caso, utiliza-se do conceito de Componentes Conexas à grade irregular. Este algoritmo permite juntar elementos adjacentes que compartilham a mesma propriedade e formar elementos maiores 
analisando a conectividade entre elementos. Neste caso, a propriedade em questão é o fato de o elemento ser parte da parede (1) ou um vazio (0). Isto permite se obter uma representação menos fracionada e mais uniforme, contribuindo para geração de um modelo tridimensional com blocos únicos.

Para compor o modelo 3D, as grades foram exportadas em formato “.obj”, considerando uma profundidade padrão para a espessura da fachada de $15 \mathrm{~cm}$ baseado na NBR 7200-1982. Feito isto, foi necessário converter o arquivo para o formato ".dxf" utilizando o software "Cloud Compare" para trabalhar com a modelagem no software Sketchup. Nesta etapa, foi necessário recuperar as informações do processo de translação para inserir cada modelo na respectiva profundidade média do plano que lhe deu origem.

\section{RESULTADOS E DISCUSSÕES}

A seguir, são apresentados os resultados obtidos aplicando a metodologia anteriormente apresentada. A metodologia foi aplicada em diversas fachadas, porém, para efeito explicativo, cabe aqui apresentar apenas uma cena. A cena escolhida conta com duas fachadas de aproximadamente 55m (Figura 8a) e 110m (Figura $8 b)$ de comprimento, respectivamente.

Figura 8 - Edificações que compõem o experimento 1.

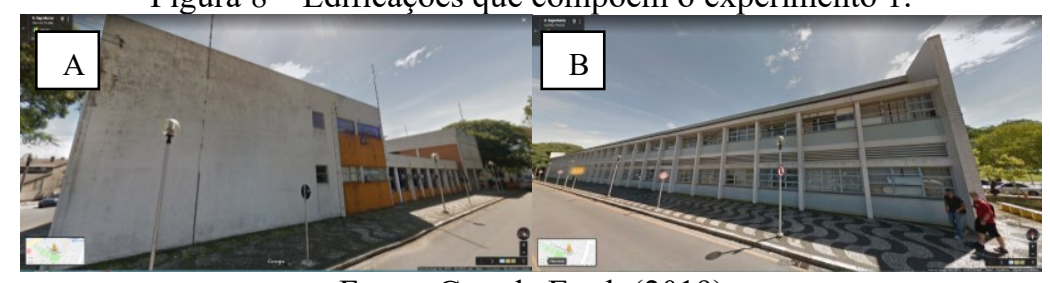

Fonte: Google Earth (2019).

O histograma de densidade sobre a nuvem de pontos foi gerado para dar subsídio na etapa de remoção da vegetação, facilitando a posterior separação das edificações. A Figura 9 se trata do resultado desta etapa para o Experimento 1. Pode-se perceber que quando se compara o histograma de frequência ao longo do eixo $\mathrm{X}$ e a nuvem de pontos da cena, os picos presentes na área em que se encontra a vegetação são menores. Isso pode ser visto na área localizada entre as linhas tracejadas em azul. Visto isto, definiu-se um limiar que diferenciasse a densidade de pontos em áreas de edificação e de vegetação, mostrado na seta em vermelho. Sendo assim, os pontos abaixo da resolução esperada para a uma edificação (limiar) foram descartados, resultando em uma nuvem de pontos filtrada, como pode ser visto na nuvem de pontos em verde a direita. Desta forma, grande parte da vegetação foi eliminada.

Figura 9 - Remoção da vegetação pela análise do histograma de densidade no eixo X.

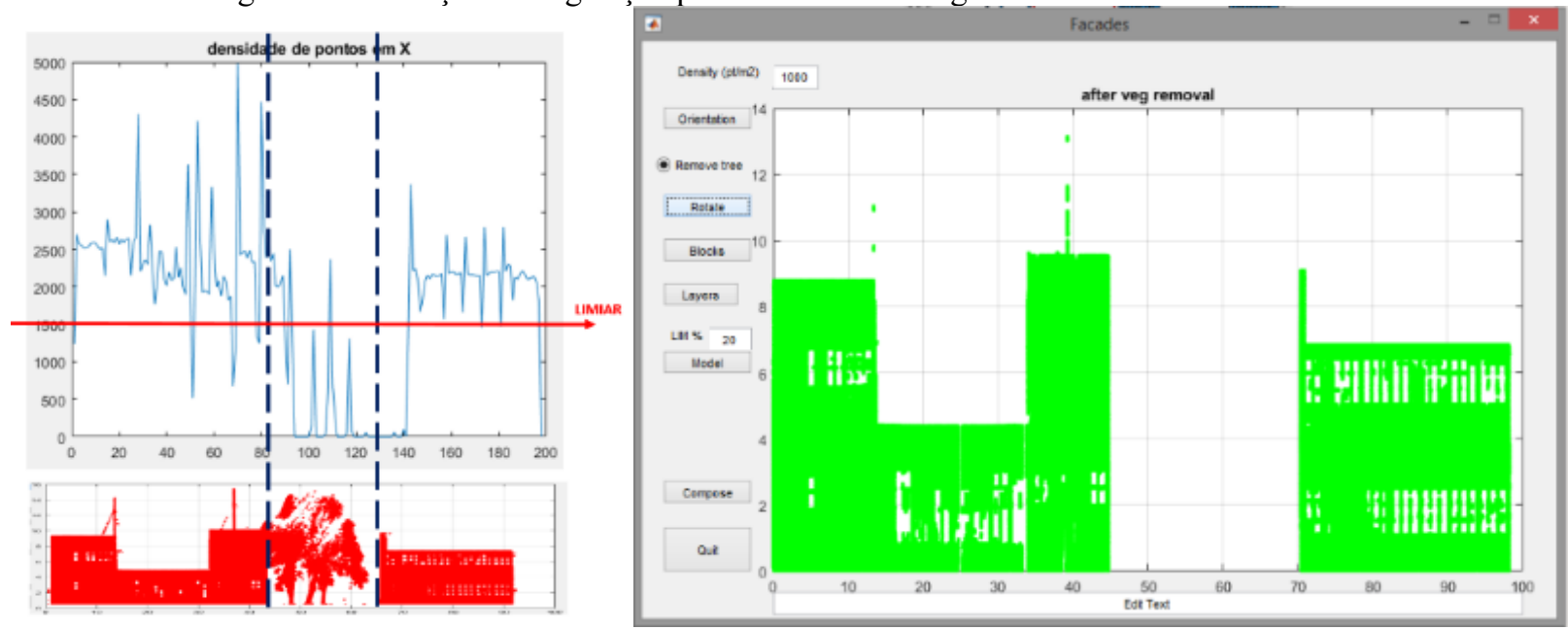

Fonte: O autor (2021). 
Logo após, tendo a nuvem de pontos apenas das edificações, foram identificados os blocos de fachada. Inicialmente foi traçado o perfil dos elementos presentes da cena para identificar as formas presentes nas edificações. Em seguida, para eliminar pontos espúrios, foram aplicadas as ferramentas de erosão e dilatação, resultando em um perfil suavizado da nuvem de pontos. Na Figura 10, tem-se o histograma da densidade em $\mathrm{X}$ da nuvem de pontos do experimento (a) e o perfil gerado após a aplicação dos filtros de morfologia matemática (b). Percebe-se que as feições relevantes da edificação foram mantidas e delimitadas de forma suave.

Figura 10 - Aplicação de morfologia matemática para suavização da nuvem.
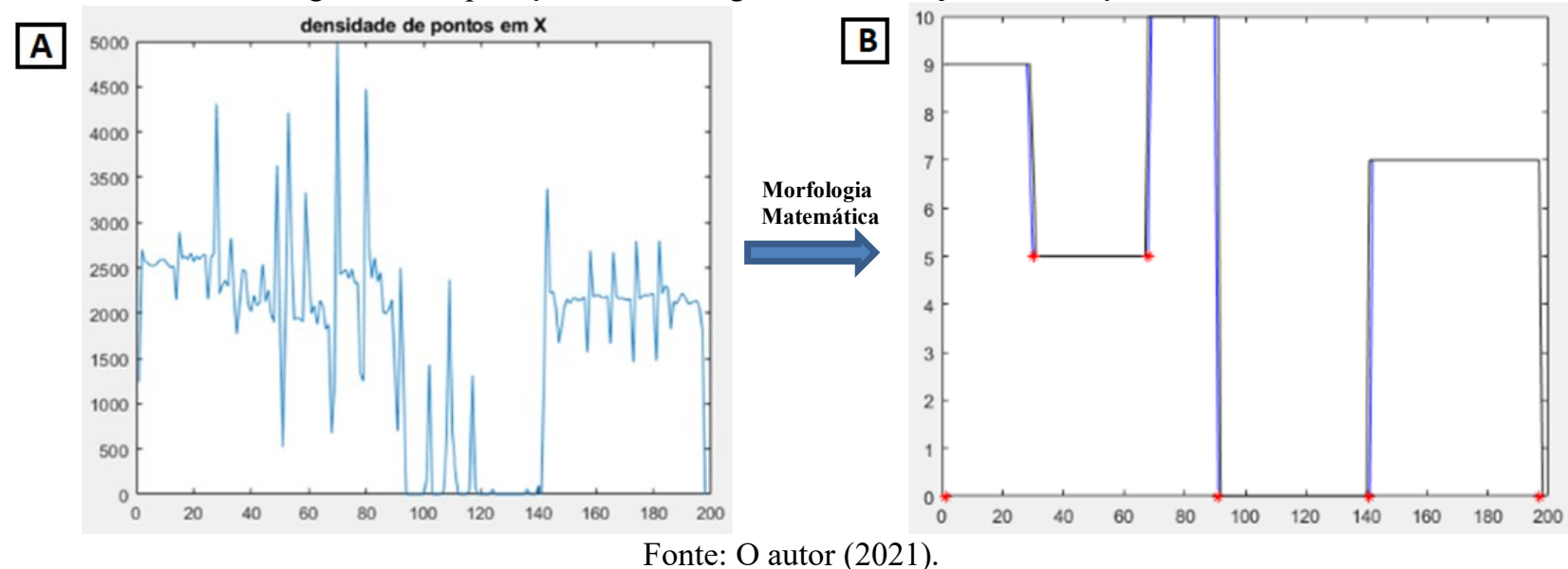

A partir dos pontos acima citados, a nuvem foi particionada em diferentes blocos de fachada, de acordo com os níveis de altura. Neste exemplo, a edificação foi dividida em quatro blocos de fachada, conforme Figura 11 , e processados separadamente nas etapas posteriores.

Figura 11 - Divisão da nuvem em blocos de fachada pela análise dos ângulos que a formam.
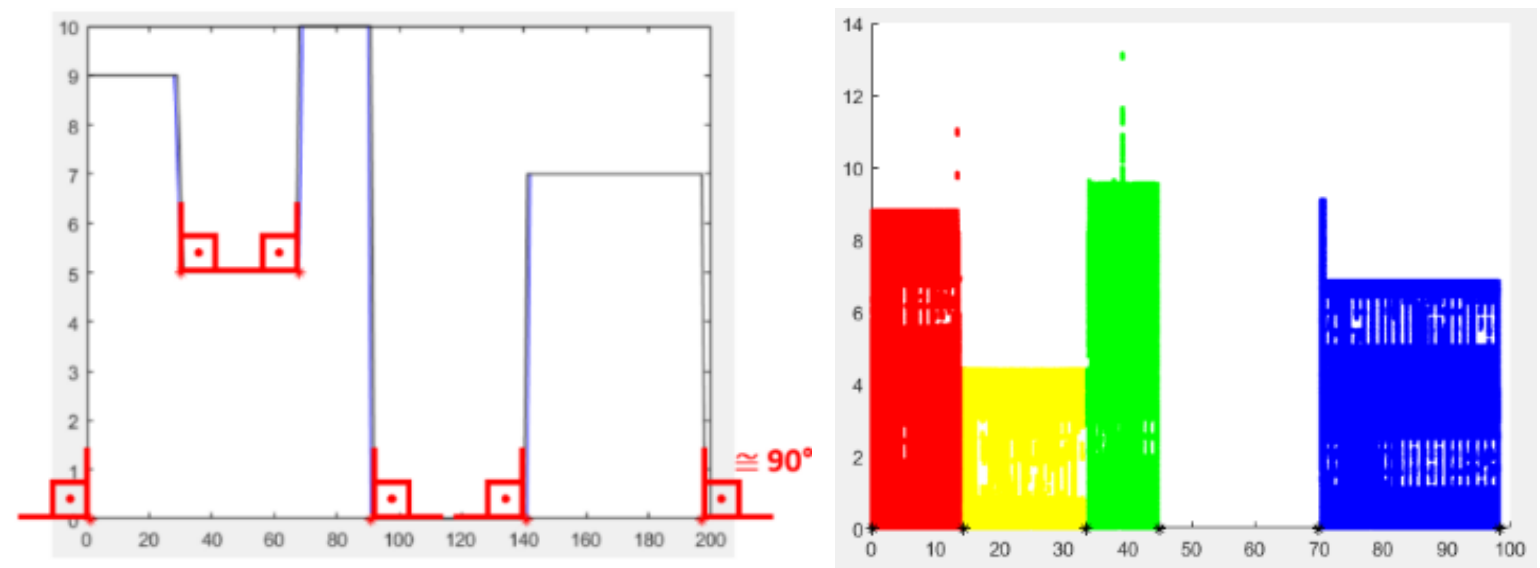

Fonte: O autor (2021).

Percebe-se que a metodologia utilizada conseguiu detectar com sucesso os blocos de fachada de acordo com as diferentes alturas. Para cada bloco foi realizada a análise dos planos que compõem a fachada em diferentes níveis de profundidade. Para isso, gerou-se o histograma de frequência de pontos no eixo Y, utilizando um passo de $0,10 \mathrm{~m}$ a fim de identificar em quais regiões haveria maior concentração de pontos, ou seja, os planos de fachadas. A escolha do passo foi condicionada ao nível de detalhamento de obras arquitetônicas, já que trabalhar com um espaçamento maior acarretaria uma representação mais genérica da edificação, desprezando elementos importantes.

No Quadro 1 são mostrados os resultados obtidos para cada bloco, podendo perceber que o algoritmo foi capaz de detectar diferentes números de planos de acordo com a especificidade da feição. Ressalta-se que 
tais cores foram escolhidas de forma aleatória para representar cada nível de fachada e não representam a real coloração da edificação.

Quadro 1 - Segmentação da nuvem de pontos em diferentes planos de fachada.

\begin{tabular}{|c|c|c|c|}
\hline Bloco & Planos de fachada & Bloco & Planos de fachada \\
\hline \multirow{3}{*}{ Bloco 1 } & & Bloco 2 & \\
\hline Bloco 3 & & & \\
\hline
\end{tabular}

Fonte: O autor (2021).

A partir dos planos de fachada, realizou-se a detecção de bordas para definição dos contornos da edificação, bem como dos elementos a ela pertencentes, como portas e janelas. Como exemplo de resultado, tem-se na Figura 12 as linhas de bordas geradas para o bloco 1, baseando-se na análise dos picos obtidos pela geração dos histogramas parciais. Vê-se que o fato da edificação possuir um padrão geométrico e regular entre portas e janelas, facilitou a definição dos contornos, já que eles foram definidos com primitivas básicas, ou seja, foram gerados vetores em forma de segmentos de retas. Ressalta-se que em fachadas que fogem a este padrão, a metodologia apresentada pode não resultar em um modelo satisfatório.

Figura 12 - Detecção das bordas a partir dos picos dos histogramas parciais em X e Y. As setas em vermelho representam picos no histograma em $\mathrm{Y}$ e as verdes em $\mathrm{X}$.

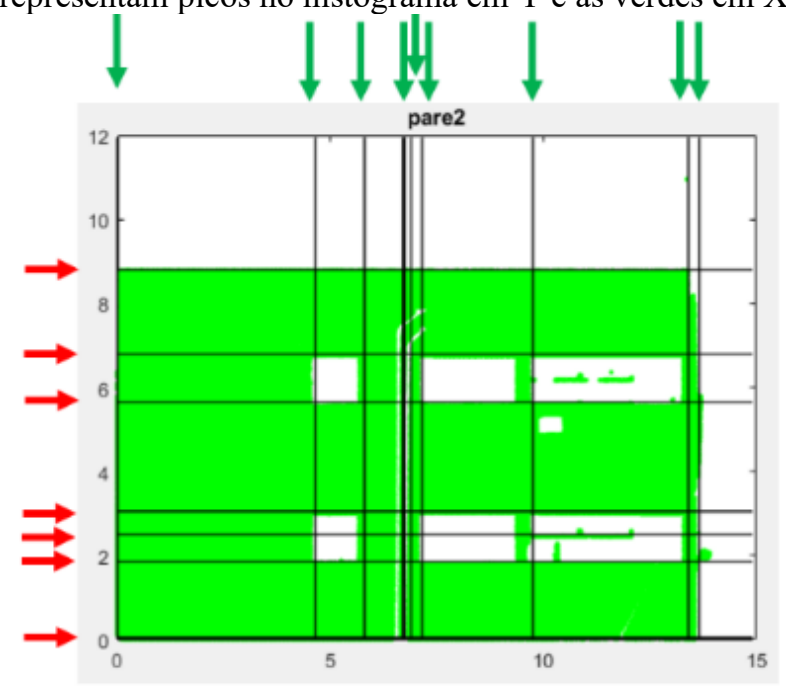

Fonte: O autor (2021).

A fim de obter resultados mais detalhados e levar em consideração as particularidades de cada faixa, foram utilizados parâmetros de detalhamento que variaram 0,05 a $0,15 \mathrm{~m}$. Dessa maneira, as faixas foram exportadas no formato ".dxf" para finalizar a modelagem no software Sketchup. Cada faixa foi inserida em seu nível de profundidade, baseando no plano médio de cada faixa. Para deixar o modelo mais refinado e regular, utilizou-se de fotos do local e visitas em campo para verificar detalhes da edificação e validar os modelos gerados. O Quadro 2 mostra o resultado desta etapa. 
Quadro 2 - Etapas de modelagem dos blocos de fachada.

\begin{tabular}{|c|c|c|c|c|c|}
\hline Bloco & Modelo Bruto & $\begin{array}{l}\text { Modelo } \\
\text { Refinado }\end{array}$ & Bloco & Modelo Bruto & $\begin{array}{l}\text { Modelo } \\
\text { Refinado }\end{array}$ \\
\hline Bloco 1 & & 日早 & Bloco 3 & & \\
\hline Bloco 2 & 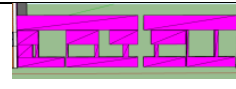 & 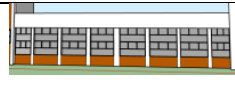 & Bloco 4 & \begin{tabular}{|l|l|} 
\\
\end{tabular} & 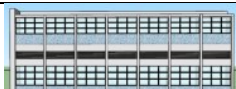 \\
\hline
\end{tabular}

Fonte: O autor (2021).

Com todos os blocos finalizados, estes foram agrupados obtendo um modelo 3D completo da fachada do edifício, conforme mostrado na Figura 13. Ressalta-se que o método proposto tem o diferencial de detectar as diversas profundidades em uma fachada, se tratando de uma vantagem em relação à modelagem apenas por fotos.

Figura 13 - Resultado da modelagem de fachadas.

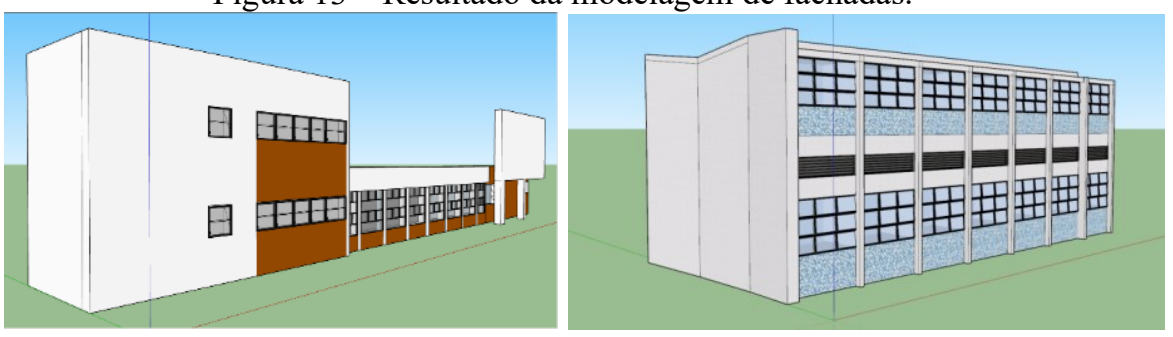

Fonte: $\mathrm{O}$ autor (2021).

Analisando o resultado obtido, vê-se esta metodologia é mais bem aplicada em edificação que possuem um padrão geométrico regular de seus elementos, tendo em vista que os resultados obtidos são blocos regulares combinados. Isso se difere, por exemplo, de metodologias que se utilizam de outras metodologias ou fontes de dados, como fotografias terrestres e aéreas.

A desvantagem de se utilizar apenas imagens como fonte de dados é a precisão do modelo obtido. Jordá (2018) salienta que a principal vantagem de utilizar pontos laser é justamente sua precisão. A qualidade da nuvem de pontos obtida por fotogrametria depende ainda de outros aspectos como a qualidade das fotos e sua cobertura e da precisão das medidas de escala utilizadas. Centeno (2004) destaca ainda a dependência de iluminação solar para captura de fotografias, já o levantamento laser depende desta condição. Vê-se ainda que uma das maiores diferenças entre o uso de imagens e o uso de LiDAR é a quantidade de dados disponíveis do objeto em estudo, no qual o laser scanner apresenta a vantagem de oferecer uma grande quantidade de pontos, efetuando o recobrimento denso da superfície.

Em Sousa et al. (2020) foi desenvolvido um modelo tridimensional a partir da combinação de imagem e levantamento topográfico, obtendo um modelo colorizado, texturizado e visualmente mais realístico. A questão é que neste estudo, o referencial geométrico foi advindo apenas de alguns pontos topográficos em face da quantidade de nuvens de pontos LiDAR utilizado no estudo aqui desenvolvido, obtido de forma mais rápida quanto comparado à topografia convencional.

Uma possível abordagem híbrida (imagem+laser) poderia garantir um modelo que mantivesse as informações advindas do laser e combinasse com os atributos obtidos por imagens (cor, material, textura, por exemplo). Uma metodologia que se assemelha à esta possibilidade pode ser vista em Deveau (2006) que conseguiu modelar objetos que não seguiam um padrão regular combinando uma nuvem TLS com imagens e utilizando o princípio sobre esses dados. 


\section{CONCLUSÃO}

Este estudo apresentou os resultados do uso nuvens de pontos obtidas com sistemas de mapeamento móvel por LiDAR, para modelagem tridimensional de fachadas e, consequentemente, de edificações em 3D. A metodologia proposta se mostrou eficiente para a reconstrução semiautomática de edificações. Aliado À uma modelagem de telhados, a metodologia pode dar subsídios para se gerar um modelo 3D num nível de detalhamento LOD3.1, onde o LoD (Level of Detail) vai do LOD0.0, representado por quadriláteros em 2D, até o LOD3.3, que seria a representação 3D de todos os detalhes da edificação. Neste o LOD 3.1 se trata do modelo que se obteve a representação tridimensional da edificação com as principais formas da fachada, suas variações de altura e profundidade e ainda a localização das portas e janelas.

É importante destacar que a metodologia utilizada garante um ganho de tempo em relação aos métodos tradicionais. Isso ocorre por dois motivos: A) o levantamento LiDAR garante a coleta de informações de uma cena de forma detalhada em poucos segundos; B) o processo proposto traz a automação da etapa de modelagem de edificações, otimizando o tempo gasto nesta fase.

O modelo resultante possui potencial para ser adaptado e utilizado em projetos que envolvam a gestão BIM, utilizando ferramentas de conversão para o formato BIM universal (.ifc). Porém, vale destacar que o levantamento das demais especificidades da edificação para geração de um modelo BIM completo, como os aspectos hidráulicos, elétricos e estruturais, devem ser obtidas por outras técnicas de sensoriamento.

Vê-se que para o sucesso da metodologia é essencial que a etapa de orientação da nuvem de pontos seja realizada, projetando os pontos nos eixos principais e tornando a fachada paralela ao eixo X. Isso garante com que se consiga recuperar as principais feições da fachada ao longo de sua profundidade. Em relação à retirada da vegetação, nota-se que é válido fazê-la de forma automática apenas quando a vegetação não estiver à frente da edificação de interesse. Caso isso aconteça, os pontos referentes à vegetação devem ser retirados manualmente, pois se mantidos, causarão ambiguidade na segmentação das feições da fachada.

Ressalta-se que a metodologia proposta apresenta resultados satisfatórios quando aplicada a edificações que seguem um padrão geométrico regular, ou seja, que possuem faces retangulares. Sendo assim, na etapa de separação por blocos de fachada, pode não ser possível separar os blocos utilizando-se a análise angular em edificações com formas curvas.

Quanto à trabalhos futuros, recomenda-se:

- Testar a aplicabilidade da técnica para diferentes realidades, tanto de formas de fachada quanto de telhado;

- Definir limiares que sejam compatíveis com grande parte das fachadas existentes, a fim de criar um algoritmo mais genérico e aplicável a diversas realidades;

- Criar uma biblioteca com blocos pré-definidos para objetos comuns em edificações, como portas e janelas, sendo estes apenas redimensionados de acordo com a fachada;

- Combinar a metodologia com outras fontes de dados, por exemplo, fotografias terrestres e aéreas, e desenvolver metodologias que extraiam a informação espectral e a correlacione a um tipo de coloração ou material a ser inserido automaticamente no modelo.

\section{Agradecimentos}

Ao Programa de Pós-Graduação em Ciências Geodésicas da Universidade Federal do Paraná, ao Instituto Lactec, pelo apoio dado para o desenvolvimento desta pesquisa e pela estrutura disponibilizada, bem como ao CNPQ devido aos benefícios concedidos pela lei no 8010/90, L.I. 12/1846474-1.

\section{Contribuição dos Autores}

O autor Luís Antônio Soares e Sousa participou da conceptualização, curadoria dos dados, análise 
formal, investigação, metodologia, software, validação e na redação do artigo. O autor Jorge A. Silva Centeno participou na conceptualização, metodologia, software, supervisão, validação e revisão e edição do artigo.

\section{Conflitos de Interesse}

Os autores declaram que não há conflito de interesse.

\section{Referências}

AMISSE, C. Modelagem 3d de telhados de edificações singulares a partir de imagens UAV para estimativa do potencial fotovoltáico e coleta de água pluvial. Dissertação (Mestrado em Ciências Geodésicas). Universidade Federal do Paraná. Curitiba, Paraná; 2016.

AWRANGJEB, M.; ZHANG, C. FRASER, C. S. Automatic reconstruction of building roofs through effective integration of Lidar and multispectral imagery. XXII ISPRS Congress. Melbourne, 2012.

BELTON, D.; LICHTI, D. Classification and segmentation of terrestrial laser scanner point clouds using local variance information. ISPRS Commission V Symposium "Image Engineering and Vision Metrology". Dresden, 2006.

BOULAASSAL, H., LANDES, T., GRUSSENMEYER, P., TARSHA-KURDI, F. Automatic segmentation of building facades using terrestrial laser data. International Symposium of Photogrammetry and Remote Sensing - ISPRS, Workshop on laser scanning 2007 and SilviLaser 2007. Finland, 2007.

BRIESE, C., PFEIFER, N. Line based reconstruction from terrestrial laser scanning data. Journal of Applied Geodesy, v. 2, pp $85-95,2008$.

CEnTENo, J. A. S; WUTKE, J. D.; Kersting, A. P. B. Comparação de geração de modelos tridimensionais usando laser scanner terrestre e restituição fotogramétrica monocular. In: Simpósio de ciências geodésicas e tecnologias da geoinformação - SIMGEO, Recife, 2004. Anais... Recife, 2004.

DEZEN-KEMPTER, E. Escaneamento 3D a laser, fotogrametria e modelagem da informação da construção para gestão e operação de edificações históricas. Gestão e Tecnologia de Projetos, v. 10, n. 2, p. $113-$ $124,2015$.

FERREIRA, F. R. Segmentação do espaço urbano por meio da tecnologia Lidar aerotransportado. Dissertação (Mestrado). Escola Politécnica da Universidade de São Paulo, São Paulo, 2014, 129p.

FONSECA, F. H.; Bertoncini, A. L. S.; LINO, A. Uso da Tecnologia LiDAR no Cadastro 3D: a determinação do Potencial Adicional de Construção. 24 SIC UDESC. Santa Catarina, 2016.

FRUEH C., SAMMON R., ZAKHOR A. Automated Texture Mapping of 3D City Models With Oblique Aerial Imagery. 3D Data Processing, Visualization and Transmission. 2nd International Symposium. Greece, 2004.

JOCHEM, A.; HOFLE, B.; WICHMANN, V.; RUTZINGER, M. \& ZIPF, A. Area-wide roof plane segmentation in airborne LiDAR point clouds. Computers, Environment and Urban Systems, v. 36, n. 1, p. 54-64, 2012.

JORDÁ, Francisco; NAVARRO, Santiago; PÉREZ, Antonio; CACHERO, Rocio; LÓPEZ, David; LERMA, José L. Close range photogrammetry and terrestrial laser scanning: high resolution texturized $3 \mathrm{~d}$ model of the chapel of the kings in the palencia cathedral as a case study. CIPA Heritage Documentation. 2018.

KABOLIZADE, M.; EBADI, H.; MOHAMMADZADEH, A. Design and implementation of an algorithm for automatic $3 \mathrm{D}$ reconstruction of building models using genetic algorithm. International Journal of Applied Earth Observation and Geoinformation, v. 19, p. 104-114, 2012.

KIM, K.; SHAN, J. Building roof modeling from airbone laser scanning data based on level set approach. ISPRS Journal of Photogrammetry and Remote Sensing, v. 66, 2011.

LAFARGE, F. Automatic building extraction from DEMs using an object approach and application to the 3D- 
city modeling. ISPRS Journal of Photogrammetry and Remote Sensing, 2002.

ORTHUBER, E.; AVBELJ, J. 3D building reconstruction from lidar point clouds by adaptive dual contouring. ISPRS Journal of the Photogrammetry, Remote Sensing and Spatial Information Sciences, v. 2, n. 3, p. 157, 2015.

PEIXOTO, E. B. A. Deteç̧ão e identificação automática de postes utilizando nuvem de pontos do sistema laser terrestre móvel. Dissertação (Mestrado em Ciências Geodésicas), Universidade Federal do Paraná, 2016.

PU, S., VOSSELMAN, M.G. Building façade reconstruction by fusing terrestrial laser points an images. Sensors, v. 9, pp 4525 - 4542, 2009.

ROTTENSTEINER, F. Automatic generation of high-quality building models from LiDAR data. IEEE Computer Graphics and Applications, v. 23, 2003.

ROTTENSTEINER, F., TRINDER, J., CLODE, S., KUBIK, K. Fusing airborne laser scanner data and aerial imagery for the automatic extraction of buildings in densely built-up areas. ISPRS Twentieth Annual Congress, pp. 512-517, 2004.

SABARIEGO, N. Modelagem de telhados a partir de nuvem de pontos lidar utilizando algoritmos genéticos. Dissertação (Mestrado em Ciências Geodésicas), Universidade Federal do Paraná, 2018.

SAMPATH, A.; SHAN, J. Segmentation and Reconstruction of polyhedral Building Roofs From Aerial LiDAR Point Clouds. IEEE Transactions on Geoscience and Remote Sensing, v. 48. 2010.

SOUSA, L. A. S.; SILVA, C. R.; VALE, M. M. B. T. Photogrammetric Survey of the Historical Church of Nossa Senhora do Rosário and São Benedito in Brazil. Journal of Civil Engineering and Architecture, 2020 .

SOUZA, D. L.; PINILLA, S. M.; TIBURCIO, T. M.S; VALLEJO, M. A. B. MILLAN, L. F. A. Processo de registro e modelagem de preexistências com uso de escaner laser terrestre. In: Simpósio Brasileiro de Qualidade do Projeto no Ambiente Construído, IV, 2015, Viçosa. Anais... Viçosa, 2015.

VOSSELMAN, G.; DIJKMAN, S. 3D Building model reconstruction from point clouds and ground plans. International Archives of the Photogrammetry and Remote Sensing, v. XXXIV-3/W4, 2001.

YANG, B.; DONG, Z. A shape-based segmentation method for mobile laser scanning point clouds. ISPRS Journal of Photogrammetry and Remote Sensing, p. 19-30, 2013.

ZHANG, R.; WU. J.; LI, G.; WANG, L. A Mixed Algorithm for Building Facade Extraction from Point Clouds. International Journal of Performability Engineering, v. 4, n. 6, p. 1119-1129, 2018.

\section{Biografia do autor principal}

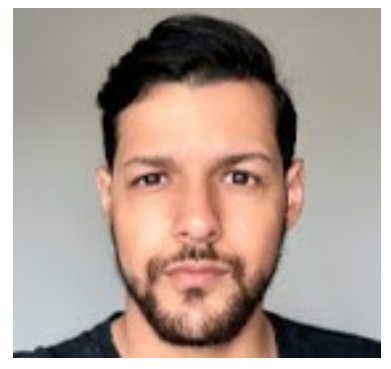

Luís Antônio Soares e Sousa, nascido em Patos de Minas-MG. Engenheiro Cartógrafo e Agrimensor formado pela Universidade Federal do Uberlândia e mestre em Ciências Geodésicas pelo Programa Pós-Graduação em Ciências Geodésicas da Universidade Federal do Paraná. Foi bolsista no LACTEC, auxiliando no desenvolvimento de pesquisas em geossoluções e possui experiência em suporte técnico e treinamentos de equipamentos topográficos e geodésicos. Hoje atua no setor de geomática na Engefoto, Curitiba-PR.

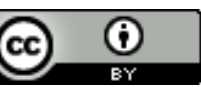

Esta obra está licenciada com uma Licença Creative Commons Atribuição 4.0 Internacional - CC BY. Esta licença permite que outros distribuam, remixem, adaptem e criem a partir do seu trabalho, mesmo para fins comerciais, desde que the atribuam o devido crédito pela criação original. 